\title{
The Susceptibility of MDR-K. Pneumoniae to Polymyxin B Plus its Nebulised Form Ver- sus Polymyxin B Alone in Critically Ill South Asian Patients
}

\author{
Md. Jahidul Hasan ${ }^{1 *}$, Raihan Rabbani², Ahmad Mursel Anam³ ${ }^{3}$, Ario Santini ${ }^{4}$, Shihan \\ Mahmud Redwanul Huq \\ ${ }^{1}$ Clinical Pharmacy Services, Square Hospitals Ltd., Dhaka, Bangladesh \\ 2 ICU and Internal Medicine, Square Hospitals Ltd., Dhaka, Bangladesh \\ 3 High Dependency Unit (HDU), Square Hospitals Ltd., Dhaka, Bangladesh \\ 4 University of Medicine, Pharmacy, Science and Technology of Targu Mures, Romania
}

\begin{abstract}
Introduction: Critically ill patients in intensive care units are at high risk of dying not only from the severity of their illness but also from secondary causes such as hospital-acquired infections. USA national medical record-data show that approximately $10 \%$ of patients on mechanical ventilation in an intensive care unit developed ventilator-associated pneumonia. Polymyxin B has been used intravenously in the treatment of multi-drug resistant gram-negative infections, either as a monotherapy or with other potentially effective antibiotics, and the recent international guidelines have emphasised the use of nebulised polymyxin B together with intravenous polymyxin $B$ to gain the optimum clinical outcome in ventilator-associated pneumonia cases caused by multi-drug resistant gram-negative infections. Methods: One hundred and seventy-eight patients with ventilator-associated pneumonia due to multidrug resistant $K$. pneumoniae were identified during the study period. Following the inclusion and exclusion criteria, 121 patients were enrolled in the study and randomly allocated to two study groups. Group 1 patients were treated with intravenous Polymyxin B plus nebulised polymyxin B $(n=64)$ and Group 2 patients with intravenous Polymyxin $B$ alone $(n=57)$. The study aimed to compare the use of Polymyxin B plus its nebulised form to polymyxin $B$ alone, in the treatment of MDR-K. pneumoniae associated ventilator-associated pneumonia in critically ill patients. Results: In Group 1, a complete clearance of $K$. pneumoniae was found in fifty-nine patients $(92.1 \% ; n=64)$ compared to forty patients $(70.1 \%, n=57)$ in the Group $2(P<0.003)$. The average time till extubation was significantly higher in Group 2 compared to Group $1(P<0.05)$. The total length-of-stay in the ICU was significantly higher in Group 2 compared to Group 1. $(\mathrm{P}<0.05)$. These results support the view that the Polymyxin $\mathrm{B}$ dual-route regime may be considered as an appropriate antibiotic therapy, in critically ill South Asian patients with ventilator-associated pneumonia.
\end{abstract}

Keywords: ventilator-associated pneumonia (VAP), multi-drug resistant $K$. pneumoniae (MDR-KP), Polymyxin B

Received: 15 July 2020 / Accepted: 23 November 2020

\section{INTRODUCTION}

Critically ill patients in intensive care units (ICU) are at high risk of dying from not only the severity of their illness but also from secondary causes such as hospitalacquired infection also known as nosocomial infection [1]. These include hospital-acquired pneumonia healthcare-associated pneumonia and ventilator-associated pneumonia each of which can lead to high rates of morbidity and mortality in hospitalised patients de- spite the administration of appropriate antimicrobial agents in appropriate care- facilities [2,3].

United States national medical record-data show that approximately $10 \%$ of patients with mechanical ventilation in an ICU experienced ventilator-associated pneumonia [4]. Ventilator-associated pneumonia can be defined as pneumonia occurred within 48-72 hours of endotracheal intubation. It is manifested by the presence of new or progressive pulmonary infiltrates, signs of systemic infection such as fever, the elevation 
of white blood cell count, changing characteristics of sputum and the presence of a virulent pathogen in the biological test samples [5].

Previously reported studies showed that $33-50 \%$ of hospital-mortality were attributable to ventilatorassociated pneumonia whereas, though this rate has declined to $9-13 \%$ in recent years because of the application of the appropriate antimicrobials and the use of suitable preventive measures [6,7].

The gram-negative Klebsiella pneumoniae is a member of the Enterobacteriaceae family. It was first isolated as an extended-spectrum $\beta$-lactamases producing organism in 1983 [8], and is considered as a serious threat to global public health [9] because of its increased resistance to most of the potential effective groups of antibiotics including beta-lactam antibiotics, aminoglycosides, and fluoroquinolones $[10,11]$. Carbapenemase-producing K. pneumoniae shows resistance against broad-spectrum carbapenems [9], and a Chinese study on an Asian population found that $K$. pneumoniae caused ventilatorassociated pneumonia in approximately $28.6 \%$ of mechanically ventilated patients [12].

In 2017, the World Health Organization cited K. pneumoniae as one of the top three emerging pathogens in the world [13]. Currently, infections including ventilator-associated pneumonia caused by multidrug-resistant K. pneumoniae (MDR-KP) have been identified as "difficult-to-treat" [14] even with last-resort potentially effective antibiotics, also known as reserve antibiotics, including polymyxins, tigecycline, aminoglycosides and carbapenems [15]. The lack of progress in the development of new antibiotics to treat life-threatening infections caused by gram-negative bacteria has made the situation more serious [13-15].

To overcome this problem, scientists are accentuating the implementation of better administration practices concerning antibiotics as well as the use of effective combinations of antibiotics [16].

Polymyxins are polypeptide antibiotics discovered in 1947 [17], and in clinical practice, the active form of polymyxin $\mathrm{B}$ and the prodrug polymyxin $\mathrm{E}$, also known as colistin, and has been adopted worldwide as the last-resort therapeutic option in gram-negative bacterial infections including MDR-KP-associated ventilator-associated pneumonia [18]. More recently, polymyxins are increasingly used in the treatment of carbapenem-resistant Enterobacteriaceae infections as they remain one of the few easily obtained antibiotics to which MDR-KP is sensitive $[18,19]$.
Over the last sixty years, polymyxin B has been used intravenously in the treatment of MDR GNB infections, either as a monotherapy or more preferably with other potentially effective antibiotics [19].

The recent International Consensus Guidelines debated the use of polymyxin B nebulisation adjunctively with intravenous polymyxin B to gain the optimum clinical outcome in ventilator-associated pneumonia cases caused by MDR-GNB [18].

The objective of this study was to compare the use of intravenous Polymyxin B concomitantly with its nebulised form with intravenous polymyxin $\mathrm{B}$ alone, in the treatment of MDR- K. pneumoniae related ventilatorassociated pneumonia in critically ill patients.

The Null hypothesis is:

There is no difference in microbial clearance in the treatment of MDR- K. pneumoniae related ventilatorassociated pneumonia, in critically ill patients when intravenous polymyxin B together with nebulised polymyxin $\mathrm{B}$ is administered compared to intravenous polymyxin $\mathrm{B}$ alone.

\section{- MATERIALS AND METHODS}

This prospective cohort study was conducted in the adult ICU of the tertiary-level Square Hospitals Ltd, Dhaka, Bangladesh, from 1 April 2018 to 30 September 2019.

\section{Inclusion criteria}

- Patient with MDR K. pneumoniae detected in endotracheal aspirates or broncho-alveolar lavage fluid in both specimens within 72 hours of ICU admission

- Age of patient $\geq 18$ years

- Less than two weeks of hospitalisation before ICU admission.

\section{Exclusion criteria}

- Patients with any other bacterial growth either in endotracheal aspirates or in broncho-alveolar lavage fluid in both specimens along with $K$. pneumoniae; growth of any gram-negative or grampositive bacteria in cultures of blood or urine samples within the preceding five days

- History of surgery within the preceding three months

- Patient with chronic or end-stage renal diseases or serum creatinine levels $>2.5 \mathrm{mg} / \mathrm{dL}$ 
- Adjusted body weight less than $40 \mathrm{Kg}$ or above 80 $\mathrm{Kg}$

- The acute physiologic assessment and chronic health evaluation (APACHE) II score of the patient above 30 during ICU admission

- Mild-to-severe hepatic impairment

- Haematological disorders

- Any history of healthcare-associated Infections within the preceding two weeks before ICU admission

- Patients with any concurrent gram-negative bacteria infections

- Coinfection with other gram-negative bacterias

Ventilator-associated pneumonia was diagnosed when pneumonia was detected within forty-eight hours of endotracheal intubation. The diagnosis was made when progressive pulmonary infiltrates were identified by chest X-ray in addition to at least two of the following symptoms: (1) Persistent fever $\geq 38^{\circ} \mathrm{C}$; (2) Leukocyte count $\geq 11,000 \backslash \mathrm{mm}^{3}$ of blood; (3) Purulent tracheobronchial secretions.

Endotracheal aspirates and broncho-alveolar lavage fluid of suspected ventilator-associated pneumonia patients were sent to the microbiology laboratory for a complete culture sensitivity report, The cut-off point $\geq 10^{6} \mathrm{CFU} / \mathrm{mL}$ of a positive quantitative culture-growth was considered for the confirmation of ventilator-associated pneumonia with contributing organisms.

The culture sensitivity testing was undertaken by micro-broth dilution using an automated microbiology system, the BD Phoenix ${ }^{\text {Tw }}$ M50 (BD Life Sciences: Diagnostics, USA.)

The susceptibility of $K$. pneumoniae isolates to an antibiotic, was confirmed by determination of the minimum inhibitory concentration, i.e. the minimum concentration at which an antibiotic prevents the growth of a particular organism.

Isolate susceptibility was undertaken according to the Clinical and Laboratory Standards Institute (CLSI) [21].

A total of 178 patients with MDR-KP- Ventilatorassociated pneumonia were identified during the study period, and this number was reduced to 121 patients after the sample inclusion and exclusion criteria were employed. These were then randomly allocated to two study groups.

Group 1. Intravenous Polymyxin B plus nebulised polymyxin B (PIVNL) ( $\mathrm{n}=64$ patients)
Group 2. Intravenous Polymyxin B (PIV) ( $\mathrm{n}=57$ patients)

The same injectable form of polymyxin B (POLY-B 500000IU Injection, Samarth Life Sciences Pvt. Ltd., India) was used in both the groups and administered over fourteen days.

On the day of admission to the ICU, the white blood cell count and the following bio-markers, c-reactive protein, procalcitonin, were measured in all patients in both Group 1 and Group 2 (Table 1), as was the renal function of patients in each group by estimating the serum creatinine level.

The dose of intravenous polymyxin B was 20,000 $25,000 \mathrm{IU} / \mathrm{kg}$ of body weight (equivalent to $2.0-2.5 \mathrm{mg} /$ $\mathrm{kg}$ ) over two hours as a loading dose, then 20,000$25,000 \mathrm{IU} / \mathrm{Kg}$ of body weight/day in two divided doses as a maintenance dose over one hour;

The dose of nebulised polymyxin B was one million units/day in two divided doses over thirty minutes through an endotracheal tube.

Dose adjustment of intravenous polymyxin B in renal impaired patients of both groups was made following standard dosing guideline [18].

In the PIVNL group, nebulised levosalbutamol was administered thirty minutes before the use of nebulised polymyxin $\mathrm{B}$.

On Day 6 post-admission, repeat endotracheal aspirate samples were obtained from each patient in both groups and sent for a review of culture sensitivity.

\section{Statistical Analysis}

Categorical variables were compared using Pearson's chi-square test or Fisher's exact test, and continuous variables were compared using the Mann-Whitney $U$ test or Student's t-test.

Multivariable logistic regression analysis was done to calculate odds ratios (ORs) with 95\% confidence intervals (CI). Continuous variables were expressed in mean (standard deviation) with 95\% CI, and categorical variables were presented as numbers (\%). KaplanMeier survival estimation was also used to evaluate 30day mortality.

The significance level was set at $\alpha=0.05$.

All the tests were two-tailed, a $p$-value $\leq 0.05$ was considered statistically significant.

Data were analysed with SPSS version 22.0 statistical software (SPSS, Chicago, IL, USA). 
Available online at: www.jccm.ro

\section{RESULTS}

All the patients of this study were of South Asian origin.

The mean (SD) age of the patients the in PIVNL group was 64.1 (16.1) years, and in the PIV group was 63.9 (14.3) years. There was no significant difference in the ages of the two groups. $(P>0.05)$.

The number of male patients was higher than female patients in both groups, 37/27 PIVNL group and 33/24 in the PIV group $(p>0.05)$.

There was no significant difference between male and female numbers in the two groups, 37/27 in the PIVNL in the group and $33 / 24$ in the PIV group $(p>0.05)$.

At the time of admission, the most common comorbidities among the patients in both groups were hypertension, diabetes mellitus, and chronic lung diseases, including asthma, chronic obstructive pulmonary disease and pulmonary fibrosis.

The mean (SD) value of APACHE II Scores in the PIVNL group and the PIV group were 18 (4.8) and 18.3 (5.5), respectively $(p<0.05)$.
The Journal of Critical Care Medicine 2021;7(1) • 31

The white blood cell count of PIVNL group was 7.5 (17.3) and that of PIV group 7.4 (14.4) $(p=0.823)$.

The mean (SD) level of serum C-reactive protein in the PIVL group was 161.7 (112.8) and 139.2 (105.3) in $\operatorname{PIV}(p=0.260)$.

The mean (SD) serum procalcitonin level in PIVNL group was 7.5 (17.3) and in the PIV group was 7.4 (14.4) $(p=0.976)$.

On admission, the mean (SD) serum creatinine level of the patients in the PIVNL group was $1.6(0.5)$ and in the PIV group was 1.5 (0.5). ( $p>0.05)$.

In the PIVNL group, a complete clearance of $K$. pneumoniae was found in fifty-nine patients (92.1\%) compared to forty patients $(70.1 \%)$ in the PIV group. $(\mathrm{OR}=0.19,95 \% \mathrm{CI}=0.06-0.58, p<0.003)$. The Null hypothesis was, therefore rejected.

In review culture sensitivity reports, in the PIVNL group, four of the sixty four patients $(6.0 \%)$ developed secondary bacterial infections whereas in the PIV group 11 of the 57 patients (19.0\%) developed second-

Table 1. Demographic and baseline characteristics of the patients

\begin{tabular}{|c|c|c|c|}
\hline \multirow{2}{*}{ Characteristics } & \multicolumn{2}{|c|}{ Variables } & \multirow{2}{*}{ p-value } \\
\hline & PIVNL group ( $n=64)$ & PIV group ( $n=57$ ) & \\
\hline \multicolumn{4}{|l|}{ Age (year) } \\
\hline Mean & $64.1 \pm 16.1$ & $63.9 \pm 14.3$ & \\
\hline Range (min-max) & $20-86$ & 20-87 & 0.950 \\
\hline \multicolumn{4}{|l|}{ Gender } \\
\hline Male & 37 & 33 & \\
\hline Female & 27 & 24 & 0.993 \\
\hline \multicolumn{4}{|l|}{ Comorbidities } \\
\hline Hypertension, n (\%) & $31(48.4)$ & $26(45.6)$ & 0.756 \\
\hline Diabetes mellitus, n (\%) & $30(46.8)$ & $27(47.3)$ & 0.957 \\
\hline Chronic lung diseases, n (\%) & $16(25)$ & $13(22.8)$ & 0.778 \\
\hline \multicolumn{4}{|l|}{ APACHE II Score (0-30) } \\
\hline Mean & $18 \pm 4.8$ & $18.3 \pm 5.5$ & \\
\hline Range (min-max) & $10-30$ & $8-30$ & 0.727 \\
\hline \multicolumn{4}{|c|}{ C-reactive protein $(<10.0 \mathrm{mg} / \mathrm{mL})$} \\
\hline Mean & 161.7(112.8) & $139.2 \pm 105.3$ & \\
\hline Range (min-max) & $11.7-393.6$ & $10.3-390.3$ & 0.260 \\
\hline \multicolumn{4}{|l|}{ Procalcitonin (<0.1 ng/mL) } \\
\hline Mean & $7.5(17.3)$ & $7.4 \pm 14.4$ & \\
\hline Range (min-max) & $0.2-114$ & $0.1-68.5$ & 0.976 \\
\hline \multicolumn{4}{|l|}{ White blood cell (4-11 K/ $\mu \mathrm{L})$} \\
\hline Mean & 19.2(7.3) & $18.9 \pm 7.3$ & \\
\hline Range (min-max) & $9.7-46.1$ & $11.1-47.6$ & 0.823 \\
\hline \multicolumn{4}{|c|}{ Serum creatinine (0.8-1.4 mg/dL) } \\
\hline Mean & $1.6(0.5)$ & $1.5 \pm 0.5$ & \\
\hline Range (min-max) & $0.6-2.3$ & $0.6-2.5$ & 0.905 \\
\hline
\end{tabular}


ary bacterial infections. $(\mathrm{OR}=0.17,95 \% \mathrm{CI}=0.05-0.59$, $\mathrm{p}<0.05)$.

The average time to extubation in the patients of PIV group was 20.5 (7.2) days which was significantly higher than the 11.7 (3.7) days in the PIVNL group $(p<0.05)$.

The total length-of-stay for patients in the ICU of PIVNL group was 16.9 (5.8) days compared to 28.7 (9.1) days in the PIV group $(p<0.05)$.

In the PIVNL group, more than $50 \%$ of patients in the PIVNL group were discharged from the ICU within 14 to 20 days. More than $60 \%$ of patients in the PIV group were discharged from the ICU after the twentyseventh day $(p<0.05)$ (Table 2$)$.

The 30-day mortality in the PIVNL group was five out of the sixty-four patients (7.8\%) and in the PIV group, nine of the fifty-seven patients (15.7\%) (OR=0.45, 95\% $\mathrm{CI}=0.14-1.43, p=0.178$ ) (Table 3 ).

Also, the Kaplan-Meier estimation showed a decrease in mortality in patients receiving intravenous Polymyxin B concomitantly with its nebulised form, compared to the intravenous administration of polymyxin B alone $(p>0.05)$ (Figure 1$)$.

Most of the patients (29.7-62.5\%) in the PIVNL group were weaned off mechanical ventilation support from the $7^{\text {th }}$ to the $20^{\text {th }}$ day of intubation, and all patient in the group were weaned off mechanical ventilation support by the fourth-week post-intubation.
The majority of patients in the PIV group were extubated from the 14 th until after the $28^{\text {th }}$-day post-intubation. Furthermore, and in this group, the time to extubation was extended to more than four weeks in $22.8 \%$ of patients (Table 3 ).

Post-extubation, in the PIVNL group, $12.5 \%$ patients were re-intubated within 21-day, and PIV group 29.8\% of patients $(p=0.089)$ (Table 3$)$.

The number of nephrotoxic adverse drug events, as assessed by the elevation of serum creatinine level above the normal range developed within forty-eight hours occurred in three of the 64 (4.6\%)patients in the PIV group, compared to three of the fifty-seven (5.2\%) patients in the PIVNL group $(P=0.773)$.

Mild bronchospasm events were developed in $6.2 \%$ of patients in the PIVNL group within 10 to 30 minutes of post-administration of nebulised polymyxin B while no bronchospasm event occurred in the PIV group $(P=0.001)$.

Dosage adjustment and the use of a bronchodilator was made immediately after the occurrence of nephron toxicity or bronchospasm in patients in both groups (Figure 2).

In this study, the K. pneumoniae isolate in the endotracheal aspirates, and broncho-alveolar lavage fluid samples were resistant to the following antibiotics: penicillin, cephalosporin, carbapenem, fluoroquinolone,

Table 2. Extubation, reintubation and hospitalisation time of the patients

\begin{tabular}{|c|c|c|c|c|c|c|}
\hline Group & $\begin{array}{l}\text { Time to extubation, } \\
\qquad \mathbf{n}(\%)\end{array}$ & p-value & $\begin{array}{c}\text { ICU length-of-stay, } \\
\text { n (\%) }\end{array}$ & $p$-value & $\begin{array}{c}\text { 21-day reintubation, } \\
\text { n (\%) }\end{array}$ & $P$ value \\
\hline \multirow{6}{*}{$\begin{array}{l}\text { PIVNL } \\
(n=64)\end{array}$} & $\geq 4$ weeks: nil & & $\geq 4$ weeks: 4 (6.25) & & \multirow{5}{*}{$8(12.5)$} & \multirow{10}{*}{$0.089 * *$} \\
\hline & $\geq 3$ weeks: 1 (1.5) & & $\geq 3$ weeks: 7 (10.9) & & & \\
\hline & $\geq 2$ weeks: 19 (29.7) & & $\geq 2$ weeks: 38 (59.4) & & & \\
\hline & $\geq 1$ week: 40 (62.5) & & $\geq 1$ week: 14 (21.9) & & & \\
\hline & <1 week: 4 (6.3) & & <1 week: 1 (1.6) & & & \\
\hline & $\geq 4$ weeks: 13 (22.8) & $0.001^{* *}$ & $\geq 1$ weeks: $36(63.2)$ & $0.002^{* *}$ & \multirow{5}{*}{$17(29.8)$} & \\
\hline \multirow{4}{*}{$\begin{array}{l}\text { PIV } \\
(n=57)\end{array}$} & $\geq 3$ weeks: 15 (26.3) & & $\geq 3$ weeks: 14 (24.5) & & & \\
\hline & $\geq 2$ weeks: 25 (43.9) & & $\geq 2$ weeks: 4 (7.0) & & & \\
\hline & $\geq 1$ week: 4 (7.0) & & $\geq 1$ week: nil & & & \\
\hline & $<1$ week: nil & & <1 week: 3 (5.3) & & & \\
\hline
\end{tabular}

Table 3. Clinical outcomes of patients with intravenous polymyxin B with or without the nebulised form.

\begin{tabular}{lcc} 
Parameter & Odds ratio $\mathbf{9 5 \%}$ confidence interval) & p-value \\
Microbial eradication & $0.19(0.06-0.58)$ & 0.003 \\
Secondary bacterial infection & $0.17(0.05-0.59)$ & 0.005 \\
30-day mortality & $0.45(0.14-1.43)$ & 0.178 \\
\hline
\end{tabular}




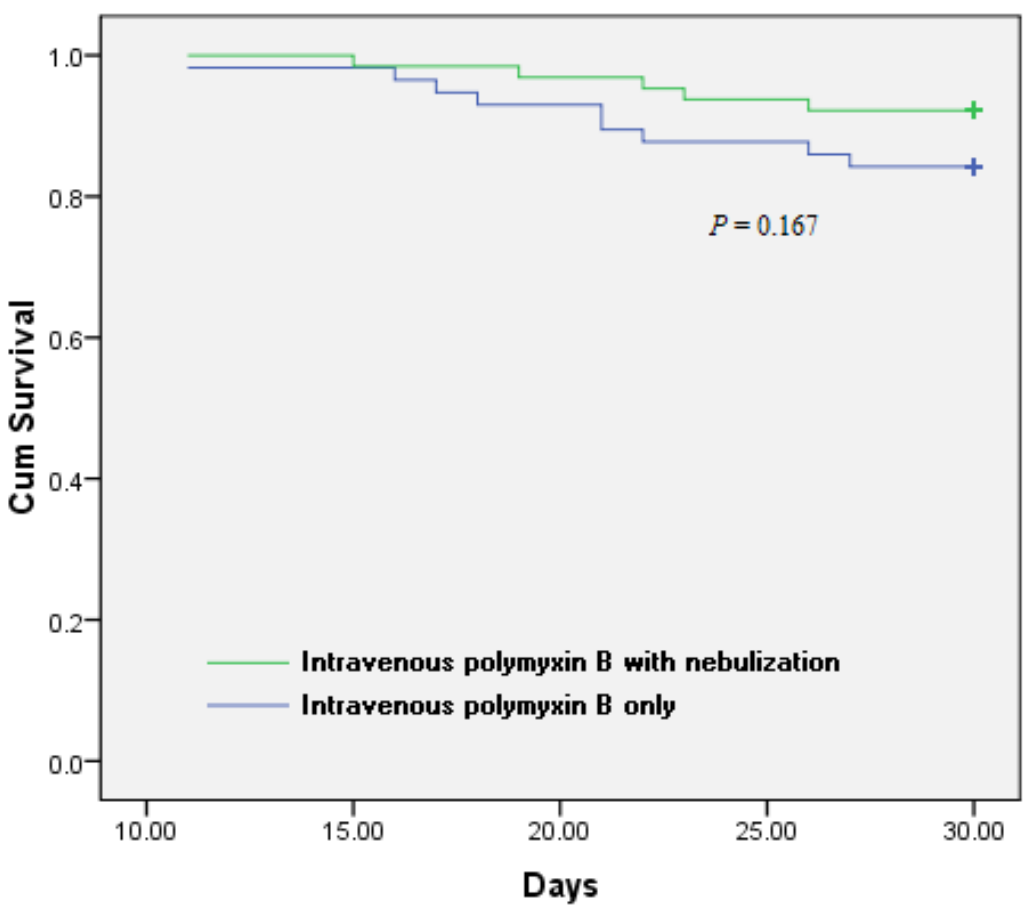

Fig. 1. Kaplan-Meier 30-day survival curve for intravenous polymyxin B with nebulization (green line) and only intravenous polymyxin B (blue line). Analysis run using Group (polymyxin B intravenously with nebulization vs intravenous polymyxin B only) as factor; death as event and time to death as time variable.

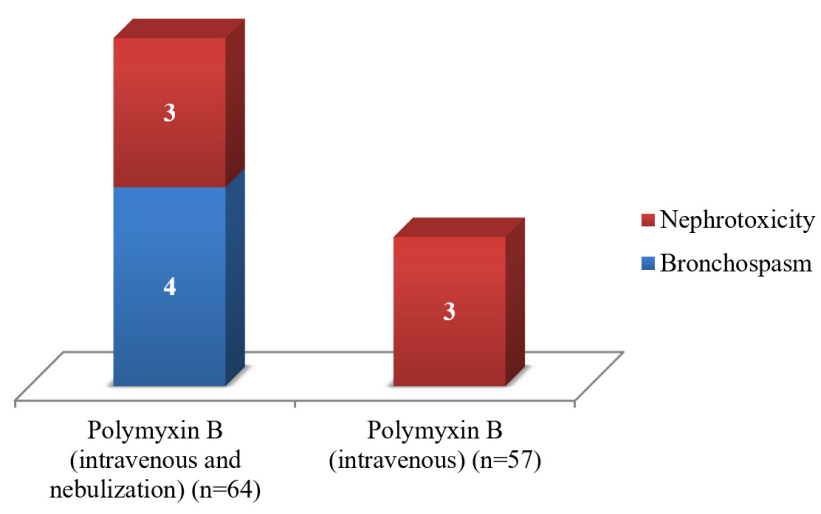

Fig. 2. Adverse events associated with polymyxin B in both the groups (polymyxin $B$ intravenously with nebulization vs intravenous polymyxin $\mathrm{B}$ alone)

and aminoglycoside but not to the polymyxins, polymyxin B and colistin.

\section{DISCUSSION}

Ventilator-associated pneumonia caused by carbapenemase-producing $K$. pneumoniae is a complicated disease with an increased chance of mortality among critically ill patients, and an effective antibiotic option to treat this serious infection is limited [12].
Polymyxin B is one of the potentially effective reserve antibiotics, still displaying promising results in severe cases [22]. In the present study, intravenous polymyxin $B$ plus nebulised polymyxin B via by the endotracheal route, showed better microbial clearance compared to intravenous polymyxin $\mathrm{B}$ alone, in the treatment of MDR K. pneumoniae-associated ventilator-associated pneumonia.

Target site-specific antibiotic therapy in life-threatening infections, including ventilator-associated pneumonia, has both promising therapeutic outcomes and related drug safety benefits [24].

Administration of polymyxin B directly to the respiratory tract is a site-specific antibiotic therapy in cases of severe lung infection, including ventilator-associated pneumonia [24]. This regime increases the chance of adequate drug concentrations in the epithelial lining fluid leading to a significant reduction in lung inflammation and restoration of lung epithelial integrity [20].

Studies have reported that a relatively high polymyxin B concentration is significantly achieved in lungs' sub-compartments after its direct aerosolised administration, and exhibits better microbial eradication compared to intravenous polymyxin B. Conversely, a single-route intravenous administration of polymyxin 
$\mathrm{B}$ in the treatment of severe pneumonia in high-risk ventilated patients of this study, was found to be less efficient in achieving microbial eradication $[25,26]$.

Following a high intravenous polymyxin B dose of 3 $\mathrm{mg} / \mathrm{kg}$ of body weight in healthy rats, one study found that the highest polymyxin B concentration was obtained in the kidneys at six hours post-administration, while a ten times lower concentration was detected in the lung tissues including the epithelial lining fluid [27]. An in-vivo study using a mouse lung infection model found that inhaled polymyxin B resulted in a high polymyxin $\mathrm{B}$ concentration in the epithelial lining fluid. In contrast, undetectable polymyxin B concentrations in the epithelial lining fluid resulted following intravenous polymyxin B alone. The concentrationtime profile analysis of polymyxin B showed that following a dose of $4.12 \mathrm{mg}$ base per $\mathrm{kg}$ of bodyweight of polymyxin $B$ resulted in an average free serum drug concentration of $1.07(0.14) \mathrm{mg} /$ litre after five minutes of intravenous administration which reduced rapidly. At the same dose, drug level in the ELF declined below the ELF limit of quantification regarding time $(<24$ hours ) which may result in a sub-optimal outcome of polymyxin B therapy, enhancement of the chance of secondary infection, and increase risk of antibiotic resistance.

Conversely, following inhalation of polymyxin B at a dose of $4.12 \mathrm{mg}$ base $/ \mathrm{kg}$ of body weight, the maximum epithelial lining fluid concentration of 107.0 (24.6) $\mathrm{mg} /$ litre, was exhibited rapidly achieved over a twelve- hours post-dose period. A large fraction of the dose was absorbed into the systemic circulation to establish a maximum plasma concentration of $0.46(0.04)$ $\mathrm{mg} /$ litre within fifteen minutes which was significantly lower than the concentration attained with intravenous administration [20].

Another study in mice, in which a high exposure of systemically administered polymyxin B against $K$. pneumoniae was given, the results showed that there was a failure to eradicate the organism [28]. These results would suggest that the intravenous administration of polymyxin B may not be a suitable therapy for the eradicating of the MDR K. pneumoniae in the lungs, especially in intubated patients due to its insufficient drug concentration in multi-segments of the lungs, and are contrary to the results of the present study.

Ventilator-associated pneumonia commonly occurred in 9 to $27 \%$ of patients undergoing mechanical ventilation support $[29,30]$, and is considered as the second most common hospital-acquired pneumonia in the ICU [31, 32]. Ventilator-associated pneumonia is accountable for approximately half of all cases of nosocomial pneumonia [33], and the occurrence rate of ventilator-associated pneumonia ranges from 1.2 to 8.5 per 1,000 ventilator days [34].

Ventilator-associated pneumonia is a significant cause of extended hospital stay in critically ill patients [35]. Patients with ventilator-associated pneumonia are highly vulnerable to developing sepsis or septic shock, as a secondary infection [35-37]. Furthermore, a study showed that the contributing factors for immunoparalysis in ventilator-associated pneumonia patients are more rampant compared to other types of bacterial infections [38].

Management of ventilator-associated pneumonia with effective antibiotic therapies [35] and appropriate sited intra-ward hygiene facilities [39], may reduce nosocomial infections and shorten the ventilation period leading to a reduced length of ICU stay. Without such measures, an extended stay in ICU may increase the chance of secondary bacterial infections leading to a higher mortality rate $[35,37]$. In the current study, we found that a shorter time to extubation, reduced length of stay in the ICU, and minimised risk of reintubation within 21-day of extubation, occurred when the infection management regime was the administration of intravenous polymyxin B plus its nebulised form, compared to the single-route intravenous polymyxin B in the treatment of ventilator-associated pneumonia linked with MDR K. pneumoniae. Also, occurrences of secondary infections and the rate of 30-day mortality declined significantly with dual-route administration compared to the single-route administration.

\section{CONCLUSIONS}

The worldwide emergence of MDR K. pneumoniae is a global health issue due to its marked levels of resistance to most of the available antibiotics, including the reserve carbapenems. To date, there is no consensus as to the ideal drug therapy for ventilator-associated pneumonia, related MDR gram-negative bacteria.

In this study, better microbial clearance, together with a decline in ICU length-of-stay and mortality, were obtained with intravenous administration of polymyxin B in combination with its nebulised form, compared to intravenous polymyxin $\mathrm{B}$ alone, in the 
Available online at: www.jccm.ro

treatment of MDR K. pneumoniae related, ventilatorassociated pneumonia.

These results support the view that the dual-route regime may be an appropriate infection management therapy, in critically ill South Asian patients with MDR $K$. pneumoniae related ventilator-associated pneumonia.

\section{- CONFLICTS OF INTEREST}

No conflicts of interest declared by the authors.

\section{- ACKNOWLEDGEMENTS}

The authors are grateful to the authority of the Square hospital for their permission to conduct this study.

\section{REFERENCES}

1. Richards MJ, Edwards JR, Culver DH, Gaynes RP. Nosocomial infections in medical intensive care units in the United States. Crit Care Med. 199;27(5):887-892.

2. Hospital-acquired pneumonia in adults: diagnosis, assessment of severity, initial antimicrobial therapy, and preventive strategies. A consensus statement, American Thoracic Society, November 1995. Am J Respir Crit Care Med. 1996;153(5):17111725 .

3. Porzecanski I, Bowton DL. Diagnosis and treatment of ventilator-associated pneumonia. Chest. 2006;130(2):597604.

4. Wang $\mathrm{Y}$, Eldridge $\mathrm{N}$, Metersky $\mathrm{ML}$, et al. National trends in patient safety for four common conditions, 2005-2011. N Engl J Med. 2014;370(4):341-351.

5. American Thoracic Society, Infectious Diseases Society of America: Guidelines for the management of adults with hospital-acquired, ventilator-associated, and healthcare-associated pneumonia. Am J Respir Crit Care Med.2005;171(4):388-416.

6. Melsen WG, Rovers MM, Koeman M, Bonten MJ. Estimating the attributable mortality of ventilator-associated pneumonia from randomised prevention studies. Crit Care Med.2011;39(12):2736-2742.

7. Melsen WG, Rovers MM, Groenwold RH, et al. Attributable mortality of ventilator-associated pneumonia: a meta-analysis of individual patient data from randomised prevention studies. Lancet Infect Dis2013;13(8):665-671.

8. Ruiz J, Gordon M, Villarreal E, Frasquet J, Sánchez MÁ, Martín M, Castellanos Á, Ramirez P. Influence of antibiotic pressure on multi-drug resistant Klebsiella pneumoniae colonisation in critically ill patients. Antimicrob Resist Infect Control 2019;8:38.
The Journal of Critical Care Medicine 2021;7(1) • 35

9. US Centers for Disease Control and Prevention. Antibiotic resistance threats in the United States, 2013. Available at: http://www.cdc.gov/drugresistance/threat-report-2013/ index.html (accessed 18 January 2020).

10. Fair RJ, Tor Y. Antibiotics and bacterial resistance in the 21st century. Perspect Medicin Chem. 2014;6:25-64.

11. Dsouza R, Pinto NA, Hwang I, et al. Panel strain of Klebsiella pneumoniae for beta-lactam antibiotic evaluation: their phenotypic and genotypic characterisation. PeerJ.2017; 5:e2896.

12. Yan $\mathrm{Q}$, Zhou M, Zou M, Liu WE. Hypervirulent Klebsiella pneumoniae induced ventilator-associated pneumonia in mechanically ventilated patients in China. Eur J Clin Microbiol Infect Dis. 2016;35(3):387-396.

13. World Health Organization. WHO Global Priority List of Antibiotic-Resistant Bacteria to Guide Research, Discovery, and Development of New Antibiotics. Available at: https:// www.who.int/medicines/publications/global-priority-listantibiotic-resistant-bacteria/en/ (accessed 20 January 2020).

14. Bassetti M, Righi E, Carnelutti A, et al. Multidrug-resistant Klebsiella pneumoniae: challenges for treatment, prevention and infection control. Expert Rev Anti Infect Ther. 2018;16(10):749-761.

15. Hsia $Y$, Lee BR, Versporten A, et al. Use of the WHO Access, Watch, and Reserve classification to define patterns of hospital antibiotic use (AWaRe): an analysis of paediatric survey data from 56 countries. Lancet Glob Health. 2019;7(7):e861-e871.

16. Aslam B, Wang W, Arshad MI, et al. Antibiotic resistance: a rundown of a global crisis. Infect Drug Resist. 2018;11:16451658.

17. Storm DR, Rosenthal KS, Swanson PE. Polymyxin and related peptide antibiotics. Annu Rev Biochem. 1977;46:723-763.

18. Tsuji BT, Pogue JM, Zavascki AP, et al. International consensus guidelines for the optimal use of the polymyxins: endorsed by the American College of Clinical Pharmacy (ACCP), European Society of Clinical Microbiology and Infectious Diseases (ESCMID), Infectious Diseases Society of America (IDSA), International Society for Anti-infective Pharmacology (ISAP), Society of Critical Care Medicine (SCCM), and Society of Infectious Diseases Pharmacists (SIDP). Pharmacotherapy. 2019;39(1):10-39.

19. Poirel L, Jayol A, Nordmann P. Polymyxins: antibacterial activity, susceptibility testing, and resistance mechanisms encoded by plasmids or chromosomes. Clin Microbiol Rev. 2017;30(2):557-596.

20. Lin YW, Zhou Q, Onufrak NJ, et al. Aerosolized Polymyxin B for Treatment of Respiratory Tract Infections: Determination of Pharmacokinetic-Pharmacodynamic Indices for Aerosolized Polymyxin B against Pseudomonas aeruginosa in a Mouse Lung Infection Model. Antimicrob Agents Chemother. 2017;61(8):e00211-17.

21. Standards of clinical and laboratory standards institute (CLSI). Performance Standards for Antimicrobial Susceptibility 
36 - The Journal of Critical Care Medicine 2021;7(1)

Testing. 30th ed. CLSI supplement M100. Wayne, PA: Clinical and Laboratory Standards Institute. 2020. Available at: https:// clsi.org/media/3481/m100ed30_sample.pdf (accessed 2 February 2020).

22. Tuon FF, Graf ME, Merlini A, et al. Risk factors for mortality in patients with ventilator-associated pneumonia caused by carbapenem-resistant Enterobacteriaceae. Braz J Infect Dis. 2017;21(1):1-6.

23. Moreira FT, Serpa Neto A. Sedation in mechanically ventilated patients-time to stay awake? Ann Transl Med. 2016;4(19):382.

24. Maxson T, Mitchell DA. Targeted Treatment for Bacterial Infections: Prospects for Pathogen-Specific Antibiotics Coupled with Rapid Diagnostics. Tetrahedron. 2016;72(25):3609-3624.

25. Hasan MJ, Rabbani R, Bachar SC. Therapeutic Specification of the Last Resort Polymyxins: An Intelligent Approach, Int J Infect. 2019;6(4):e96828.

26. Hasan MJ. Polymyxins Nebulization over Intravenous Injection: Pharmacokinetics and Pharmacodynamics-Based Therapeutic Evaluation. J Pharm Res Int. 2019;25(4):1-10.

27. Manchandani P, Zhou J, Ledesma KR, et al. Characterisation of Polymyxin B Biodistribution and Disposition in an Animal Model. Antimicrob Agents Chemother. 2015;60(2):10291034.

28. Landersdorfer CB, Wang J, Wirth $\mathrm{V}$, et al. Pharmacokinetics/ pharmacodynamics of systemically administered polymyxin $B$ against Klebsiella pneumoniae in mouse thigh and lung infection models. J Antimicrob Chemother. 2017;73(2):462468.

29. American Thoracic Society, Infectious Diseases Society of America. Guidelines for the management of adults with hospital-acquired, ventilator-associated, and healthcare-associated pneumonia. Am J Respir Crit Care Med.2005;171(4):388-416.
Available online at: www.jccm.ro

30. Chastre J, Fagon JY. State of the art: ventilator-associated pneumonia. Am J Respir Crit Care Med.2002;165(7):867-903.

31. Hunter JD. Ventilator-associated pneumonia. BMJ.2012;344:e3325.

32. Kalanuria AA, Ziai W, Mirski M. Ventilator-associated pneumonia in the ICU. Crit Care. 2014;18(2):208.

33. Vincent JL, Bihari DJ, Suter PM, et al. The prevalence of nosocomial infection in intensive care units in Europe. Results of the European Prevalence of Infection in Intensive Care (EPIC) Study. EPIC International Advisory Committee. JAMA. 1995;274(8):639-644.

34. Skrupky LP, McConnell K, Dallas J, Kollef MH. A comparison of ventilator-associated pneumonia rates as identified according to the National Healthcare Safety Network and American College of Chest Physicians Criteria. Crit Care Med.2012;40(1):281-284.

35. Heredia-Rodríguez M, Peláez MT, Fierro I, et al. Impact of ventilator-associated pneumonia on mortality and epidemiological features of patients with secondary peritonitis. Ann Intensive Care. 2016; 6(1):34.

36. Singer M, Deutschman CS, Seymour CW, et al. The Third International Consensus Definitions for Sepsis and Septic Shock (Sepsis-3).JAMA.2016;315(8):801-810.

37. Zhao GJ, Li D, Zhao Q, et al. Incidence, risk factors and impact on outcomes of secondary infection in patients with septic shock: an 8-year retrospective study. Sci Rep. 2016; 6:38361.

38. Christaki E. Host immune response in sepsis due to ventilatorassociated pneumonia: How is it different? Crit Care. 2009;13(6):1009.

39. Ture Z, et al. A Comparison of Nosocomial Infection Density in Intensive Care Units on Relocating to a New Hospital. J Crit Care Med. 2020;6(3):175-80. 